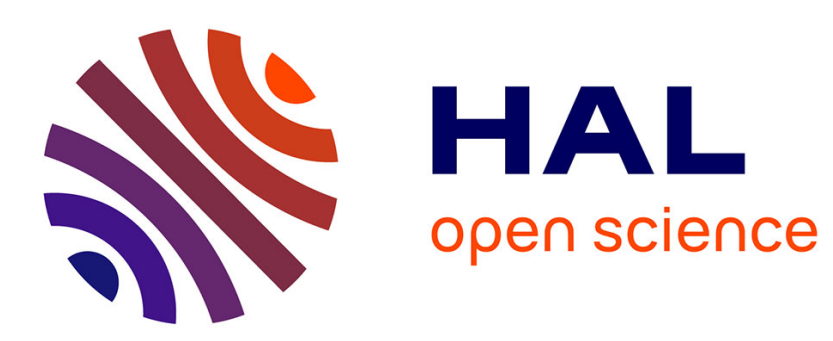

\title{
Erasure of nanopores in silicate glasses induced by femtosecond laser irradiation in the Type II regime
}

Maxime Cavillon, Yitao Wang, Bertrand Poumellec, François Brisset, Matthieu Lancry

\section{- To cite this version:}

Maxime Cavillon, Yitao Wang, Bertrand Poumellec, François Brisset, Matthieu Lancry. Erasure of nanopores in silicate glasses induced by femtosecond laser irradiation in the Type II regime. Applied physics. A, Materials science \& processing, 2020, 126, 10.1007/s00339-020-04062-8 . hal-03093803

\section{HAL Id: hal-03093803 https://hal.science/hal-03093803}

Submitted on 4 Jan 2021

HAL is a multi-disciplinary open access archive for the deposit and dissemination of scientific research documents, whether they are published or not. The documents may come from teaching and research institutions in France or abroad, or from public or private research centers.
L'archive ouverte pluridisciplinaire HAL, est destinée au dépôt et à la diffusion de documents scientifiques de niveau recherche, publiés ou non, émanant des établissements d'enseignement et de recherche français ou étrangers, des laboratoires publics ou privés. 
1 Title: Erasure of nanopores in silicate glasses induced by femtosecond laser irradiation in

2 the Type II regime

3 Authors : Maxime Cavillon ${ }^{\dagger, *}$, Yitao Wang ${ }^{\dagger}$, Bertrand Poumellec ${ }^{\dagger}$, François Brisset ${ }^{\dagger}$, and

4 Matthieu Lancry $^{\dagger}$

5 Institut de Chimie Moléculaire et des Matériaux d'Orsay (ICMMO/SP2M/MAP),

6 Université Paris-Saclay, CNRS, 91405 Orsay Cedex, France

7 * corresponding author: maxime.cavillon@ universite-paris-saclay.fr

Abstract: Optical devices fabricated by femtosecond (fs) laser within the Type II regime are of interest for high temperature applications $\left(>800^{\circ} \mathrm{C}\right.$ ). fs-Type II regime is characterized by the formation of self-organized nanogratings, which are composed of regularly spaced porous nanolayers with nanopores having a typical size of a few tens of $\mathrm{nm}$. In this work, we first investigate the evolution of the nanopores size distribution as a function of fs-laser writing speed and pulse energy, as well as a function of annealing temperature after fs-laser irradiation. Then, the thermal stability of such nanopores is numerically investigated through the use of the Rayleigh-Plesset (R-P) equation, and is compared with experimental data. The R-P equation provides insights into the temperature range at which the nanopores would ultimately collapse, serving as a design tool for future high temperature fs-Type II based devices. The key role of glass viscosity and nanopore size diameter on the overall thermal stability is also discussed.

Keywords: femtosecond laser; nanogratings; silicate glasses; thermal stability

\section{Introduction}

Optical devices capable to withstand and operate at high temperatures $\left(>800^{\circ} \mathrm{C}\right)$ for a long period of time (e.g. hundreds of hours) are attractive for many applications, including aircraft engine monitoring, fuel bed combustors, long lifetime optical data storage or fiber lasers[1-3]. Within the tools at one's disposal to fabricate devices in both fiber and bulk glass materials, femtosecond laser-direct writing (FLDW) is particularly interesting. Indeed, FLDW is a versatile technique that enables high peak powers to induce local threedimensional (3D) modifications inside the glass subtract, due to the nonlinear absorption processes involved during the laser light - matter interaction. One of the unique features of FLDW is the possibility to induce self-organized nanogratings, within the so-called Type II 
regime [4]. In addition, nanogratings have been reported in a variety of glasses, including silica, germanates, silicates, borosilicates $[5,6]$.

In silica, these nanogratings can survive beyond $1000{ }^{\circ} \mathrm{C}$ for several hours [7], and are composed of regularly spaced porous nanolayers (typically few tens of nm thickness, with a period of about $300 \mathrm{~nm}$ ). The latter are filled with nanopores having their size and number being a function of the laser parameters as well as the glass material [8]. Upon an increase in temperature, Type II regime laser-induced modifications would yield changes in the glass structure (e.g., point defects, stress, specific volume) and in the related physical properties (e.g., refractive index, birefringence, light absorption and scattering). Such modifications will ultimately dictate the response and the lifetime of a fabricated optical device (e.g., a grating used as a sensor [2] or a dot used for 5D optical data storage [3].

Thus, it is critical to understand what are the underlying mechanisms that drive the evolution of these aforementioned modifications at high temperatures. The annealing of fslaser induced nanogratings yield to some modifications, at different temperature ranges, which have previously been investigated and reported. For instance, the presence of laser induced defects, such as non-bridging oxygen hole centers (NBOHC), fully erased at $600^{\circ} \mathrm{C}$ within a few minutes [9]. Additionally, in silica the typically large amount of 3- and 4membered rings, characteristic of densification and the presence of an intense compressive stress induced by Type II laser irradiation, is found to decrease and to anneal away within the $800^{\circ} \mathrm{C}-900^{\circ} \mathrm{C}$ temperature range $[9,10]$. Moreover, the stress field associated with the formation of nanogratings (see for instance Ref. [11]) is typically relaxed slightly below or at the glass transition temperature $\left(\square 1100^{\circ} \mathrm{C}\right.$ for silica) $[10,12,13]$. Ultimately, the fsinduced modifications correspond to a permanently decomposed glass with nanopores embedded inside it $[14,15]$. As a note, the presence of defects in the glass, as discussed above, is partly associated with the dissociation of $\mathrm{SiO}_{2}$ into $\mathrm{SiO}_{2(1-\mathrm{x})}+\mathrm{xO}_{2}$ [14]. The Raman peak intensity, centered at $1556 \mathrm{~cm}^{-1}$ in Ref. [14], is characteristic of free $\mathrm{O}_{2}$ inside the glass nanopores [16]. Increasing the temperature in the $300^{\circ} \mathrm{C}-600^{\circ} \mathrm{C}$ range causes bleaching of defects, which would yield a decrease of the $\mathrm{O}_{2}$ pressure. The signature of free $\mathrm{O}_{2}$ is detected up to $\square 600{ }^{\circ} \mathrm{C}$ using Raman spectroscopy (see Figure 3.14 of Ref. [17]), and agrees well with the aforementioned defect bleaching.

In this work, we first investigate the nanopores size distribution, in silica glass, for different writing conditions (speed and pulse energy), as well as the erasure of the nanopores through heat treatments at different temperatures. Variations in the average 
nanopore size diameter are observed [18]. Following this, the thermal stability of the laser induced nanopores is investigated using the Rayleigh-Plesset (R-P) equation [19], assuming the nanopores to have a spherical shape. In practice, the nanopores can take the form of ellipsoids or more complex shapes [20, 21]. However, this work intends to set the basis for future work to include these difficulties and therefore only spherical particles is considered.

The role played by both the glass material (including silica - Infrasil and PCVD types, Borofloat $33-81 \% \mathrm{SiO}_{2}-\mathrm{B}_{2} \mathrm{O}_{3}-\mathrm{N}_{\mathrm{a} 2} \mathrm{O}-\mathrm{Al}_{2} \mathrm{O}_{3}$, and Ultra low expansion-ULE glass - glass Ultra Low Expansion $93 \% \mathrm{SiO}_{2}-\mathrm{TiO}_{2}$ ) and the initial nanopore size diameter on the overall thermal stability of the fs-induced nanogratings are modeled using the R-P equation, and compared with experimental data. This work is aimed to provide preliminary insights on the ultimate stability of the nanopores present in the glass. This may be used as a means to ease the selection of glass material and laser parameters to enhance thermal stability in future optical designs operating in a high temperature environment.

\section{Materials and Methods}

First, the nanopore size distribution within the porous regions is investigated for three different glass samples (labeled S1, S2 and S3), which are reported in Table 1, along with the different laser irradiation conditions. In S1 sample the writing speed is varied from 0.01 $\mathrm{mm} \mathrm{s}^{-1}$ to $10 \mathrm{~mm} \mathrm{~s}^{-1}$. In S2, the pulse energy is varied from $0.2 \mu \mathrm{J}$ to $1.0 \mu \mathrm{J}$, and in S3 Type II laser-induced modifications are consecutively annealed for 30 minutes at $1000^{\circ} \mathrm{C}$, $1100^{\circ} \mathrm{C}$, and $1200^{\circ} \mathrm{C}$. In order to investigate the nanopores and their size distribution, each sample was cleaved perpendicularly to the laser light polarization direction (for instance see Ref. [1]) and imaged by a scanning electron microscope (FEG-SEM Zeiss Supra 55 VP).

Table 1 Glass samples and laser irradiation conditions investigated for nanopore size distribution characterization

\begin{tabular}{cccc}
\hline Materials & Silica SuprasilCG & Silica PCVD & $\begin{array}{c}\text { Silica } \\
\text { suprasilCG }\end{array}$ \\
\hline $\begin{array}{c}\text { Parameters Sample } \\
\text { Labelling }\end{array}$ & $\mathbf{S 1}^{1}$ & $\mathbf{S 2}$ & $\mathbf{S 3}$ \\
\hline $\begin{array}{c}\text { Pulse energy }(\mu \mathrm{J}) \\
\text { Writing speed, } v\left(\mathrm{~mm} \mathrm{~s}^{-1}\right)^{2}\end{array}$ & $0.01 ; 0.1 ; 1 ; 10$ & $0.2 ; 0.4 ;$ and 1.0 & 1.0 \\
Repetition rate, $f(\mathrm{kHz})$ & 100 & 0.1 & 0.1 \\
\hline
\end{tabular}




\begin{tabular}{cccc}
\hline Number of pulses per $\mu \mathrm{m}$ & 10,$000 ; 1,000 ; 100 ; 10$ & 1,000 & 1,000 \\
Wavelength $(\mathrm{nm})$ & 1030 & 1030 & 1030 \\
Pulse duration (fs) & 250 & 300 & 300 \\
Numerical aperture, NA & 0.6 & 0.6 & 0.6 \\
Focusing depth $(\mu \mathrm{m})$ & - & 1300 & 1300 \\
Configuration $^{3}$ & - & $/ /$ & $/ /$ \\
\hline
\end{tabular}

90

${ }^{1}$ This sample is the same as in Ref. [8].

${ }^{2}$ Varying the laser writing speed $v$ with a constant repetition rate $f$ is equivalent to changing the pulse density $v / f$, or the "number of pulses per $\mu \mathrm{m}$ " as defined in the table.

3 // or ?: Laser polarization parallel (//) or perpendicular (?) to the writing direction.

Following the investigation of the nanopore sizes, a numerical approach, using the Rayleigh-Plesset (R-P) equation, is undertaken to simulate the erasure of the nanopores during the thermal annealing. The R-P equation is derived from the Navier-Stokes equation and physically describes the evolution of a spherical bubble inside an incompressible Newtonian fluid. The R-P equation takes the following form [19, 22, 23]:

$$
\frac{\Delta P}{\rho}=R_{\text {pore }} \frac{d^{2} R_{\text {pore }}}{d t^{2}}+\frac{3}{2}\left(\frac{d R_{\text {pore }}}{d t}\right)^{2}+\frac{4 \eta(T)}{\rho R_{\text {pore }}} \frac{d R_{\text {pore }}}{d t}+\frac{2 S}{\rho R_{\text {pore }}} .
$$

In this equation, $\Delta P$ is the pressure difference (in $\mathrm{Pa}$ ) between the inside of the nanopore (supposed spherical) and far away from it, $\rho$ is the glass density (in $\mathrm{kg} \mathrm{m}^{-3}$ ), $R_{\text {pore }}$ the radius (in $\mathrm{m}$ ) of the spherical nanopore, $\mathrm{t}$ the time (in $\mathrm{s}$ ), $\eta(\mathrm{T}$ ) the glass viscosity (in Pa.s), and $S$ is the surface energy (around $0.3 \mathrm{~J} \mathrm{~m}^{-2}$ for silica [24]). Both $\rho$ and $S$ are set independent to the temperature for sake of simplicity. The viscosity is extrapolated from glass manufacturer datasheets (Borofloat33, SuprasilCG, Infrasil and PCVD) and from Ref. [8] for ULE glass, using a Vogel-Tammann-Fulcher (VTF) law in the form $\log (\eta)=A+B /(T-T 0)$. Initial conditions are $R_{\text {pore }}$ (at $\left.t=0\right)=R_{0}$ and $\mathrm{dR}_{\text {pore }} / \mathrm{dt}$ (at $t=0$ ) $=0$. The equation is solved numerically using an ordinary differential equation (ODE) built-in solver from Matlab software. This work only intends to provide preliminary understandings on the ultimate stability of nanopores using the R-P equation. Therefore, several assumptions and simplifications are made. First, we set $\Delta P=0$, as the decrease in $\mathrm{O}_{2}$ concentration inside the nanopore, due to bleaching of defects as described in the introduction below $600^{\circ} \mathrm{C}$. It would result in a rather small $\mathrm{O}_{2}$ pressure, which will be subsequently neglected throughout the paper. The R-P equation is used to compute the nanopore size evolution for different glasses, 
114 including pure silica (PCVD, Infrasi1301), Borofloat33 and ULE, which have been reported 115 in Ref. [8]. In these experiments, the thermal stability of Type II nanostructures is 116 investigated through step isochronal measurements $\left(\Delta t=30\right.$ minutes, $\Delta T$ typ. $25^{\circ} \mathrm{C}$ or $\left.50^{\circ} \mathrm{C}\right)$ 117 and by monitoring the retardance induced by the birefringent nanogratings. The Retardance $118(R)$ is defined as the product of the Birefringence $(B)$ with the length $(L)$ of the birefringent 119 object $(R=B \times L)$. In Ref. [8], the aforementioned $R(\Delta t, T)$ values measured at room 120 temperature after each annealing step are normalized relative to the initial (not annealed) $R(t$ $121=0,20^{\circ} \mathrm{C}$ ) value. In this work, the experimental data are normalized with respect to the $R$ 122 value at the temperature above which the retardance experiences a steep decay rate (e.g., $T=$ $1231225^{\circ} \mathrm{C}$ for Infrasil301 in figure 5a in Ref. [8]). The latter normalization enables to 124 investigate only the last step of nanograting erasure.

125 In order to compare the computed results from the R-P equation with the experimental 126 normalized $R$ values, the nanopore size diameter (from R-P equation) is converted to a 127 normalized retardance value following the procedure described below. First, we calculate the 128 average refractive index of the porous nanolayer $\left(n_{p l}\right)$ using the Maxwell-Garnet equation 129 [25]:

$$
n_{p l}=\sqrt{n_{G}^{2} \frac{n_{G}^{2}+\frac{(1+2 F F)}{3}\left(n_{\text {pore }}^{2}-n_{G}^{2}\right)}{n_{G}^{2}+\frac{(1-F F)}{3}\left(n_{\text {pore }}^{2}-n_{G}^{2}\right)}} .
$$

130 In the above equation, $\mathrm{n}_{\mathrm{G}}$ is the glass refractive index (taken as 1.4599 , i.e., index of silica at $131551 \mathrm{~nm}$ ), which is assumed independent of temperature (small dn/dT of $\square 10 \times 10^{-6} \mathrm{~K}^{-1}[26]$ ). 132 This value is taken equal both inside the porous nanoplanes (i.e., around the nanopores) and 133 between the porous nanoplanes. This simplification is acceptable in the conditions of this 134 work, as we principally focus on the erasure of the nanopores for temperatures at, or above, 135 the glass transition temperatures of the glass materials investigated. Therefore, the 136 contributions to the refractive index due for instance by the presence of a stress field or a 137 permanent densification of the glass are annealed away.

$138 F F$ is the filling factor, defined here as the relative volume taken by the nanopores per unit 139 volume of porous nanolayer (labeled $V$ ), and $n_{\text {pore }}$ is the nanopore refractive index, taken 140 equal to 1 . The filling factor is related to the averaged nanopore radius $\left(R_{\text {pore }}\right)$ through the 141 following equation: 


$$
F F=N \frac{4 \pi R_{\text {pore }}^{3}}{3 V}
$$

Here $N$ is the number of nanopores (with an averaged nanopore radius $R_{\text {pore }}$ ) per unit volume $V$ of porous nanolayer. A 2D filling factor (i.e., taking the area taken by the nanopores per unit surface of porous nanolayer) can be determined from SEM micrographs and is usually in the order of $20 \%-40 \%$ [18]. The latter (labeled $F F_{2 D}$ ) will be used in section 3.4 . When discussing the normalized retardance in section 3.23 , the $F F$ defined in Equation 3 is computed. Additionally, in this work, the refractive index of the glass in between the porous nanolayers is set equal to $\mathrm{n}_{\mathrm{G}}$ as in Ref. [18]. Hence, the birefringence $B=n_{o}-n_{e}$, where $n_{o}$ and $n_{e}$ are the refractive indices of the ordinary and extraordinary axes, respectively, is calculated from [7, 18]:

$$
B=n_{o}-n_{e}=\sqrt{\left[1-\frac{\delta}{\Lambda}\right] n_{G}^{2}+\frac{\delta}{\Lambda} n_{p l}^{2}}-\left[\sqrt{\left.\frac{\left[1-\frac{\delta}{\Lambda}\right]}{n_{G}^{2}}+\frac{\frac{\delta}{\Lambda}}{n_{p l}^{2}}\right]^{-1}} .\right.
$$

$142 \Lambda$ is the average spacing between nanolayers, $\delta$ is the porous nanolayer thickness, and $(\Lambda-\delta)$

143 is the interlayer thickness. Finally, the linear retardance can be calculated using the 144 expression $R=B \times L$ as defined above.

145

146

147

148

149

150

151

152

153

154

155

156

\section{Results}

\subsection{Experimental observation of the evolution of the nanopore size in nanogratings Impact}

\section{of laser writing conditions on the evolution of nanopores size}

The impact of the writing speed in the nanopore distribution is depicted in Fig. 1, where SEM micrographs, nanopore size distribution, 2D filling factor and averaged nanopore size-diameter as a function of writing speed, are displayed (using Sample S1). We observed that the average nanopore diameter increases when writing speed increases (from $53 \mathrm{~nm}$ to $77 \mathrm{~nm}$ from $0.01 \mathrm{~mm} \mathrm{~s}^{-1}$ to $10 \mathrm{~mm} \mathrm{~s}^{-1}$, respectively, corresponding to a $\square 45 \%$ increase). As the writing speed increases, the spread of the nanopore diameter distribution also becomes larger (e.g. standard deviation is increased from $13 \mathrm{~nm}$ to $34 \mathrm{~nm}$ between $0.01 \mathrm{~mm} \mathrm{~s}^{-1}$ and $10 \mathrm{~mm} \mathrm{~s}^{-1}$ ). While the average nanopore size is increased when speed is increased, the $2 \mathrm{D}$ filling factor comparatively decreases. The evolution of $F F_{2 D}$ observed in 
Fig. 1 is qualitatively in agreement with retardance values reported in Ref. [8]. However, it is worth pointing out that other contributions factor into the measured retardance value,

159 such as laser track length, porous nanolayer thickness or periodicity.

(a)
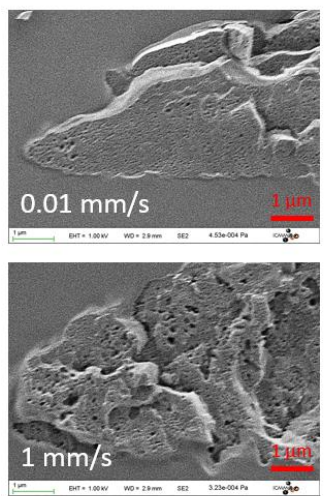

(b)

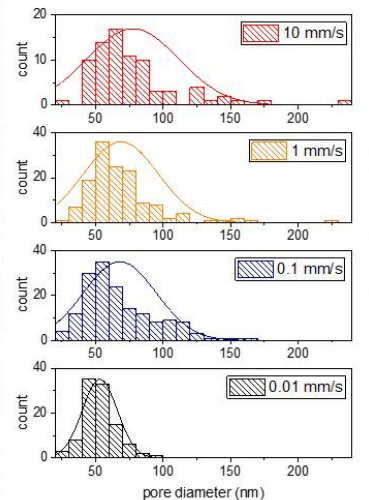

(c)

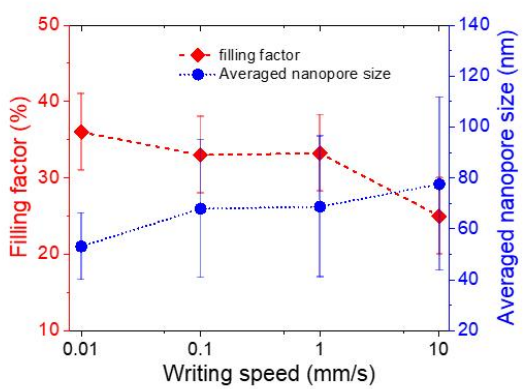

160

161

162

163

164

165

166

167

168

169

170

171

172

173

174

175

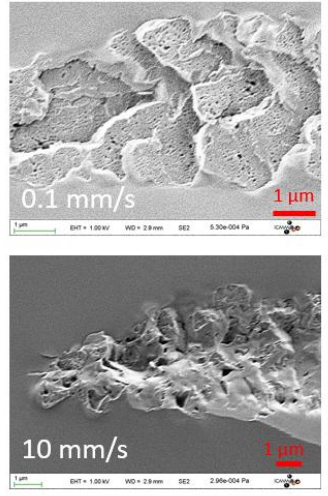

Fig. 1 Effect of the writing speed on nanopore size, distribution and filling factor, using Sample S1. (a) Scanning electron micrographs of the porous regions for different writing speeds; (b) Nanopore size diameter distributions with respect to the writing speed; (c) Evolution of the 2D porosity filling factor and the average nanopore size diameter as a function of writing speed. The lines are here only as guide-to-the-eye

The evolution of the nanopore size and distribution, as presented in Fig. 1 with respect to varying writing speed conditions, is now investigated through the variation of the laser pulse energy, everything else being set constant (from Sample S2). The results are reported in Fig. 2. An increase of the pulse energy is associated with an increase in the nanopore size diameter, which exhibits an almost linear rate within the investigated interval ( $\square 8.6 \mathrm{~nm}$ diameter increase per $\mu \mathrm{J})$. There is almost no evolution of the standard deviation $(5.3 \mathrm{~nm}$ and $5.9 \mathrm{~nm}$ for $0.2 \mu \mathrm{J}$ and $1.0 \mu \mathrm{J}$, respectively). However, as opposed to the results from Fig. 1a, the increase in energy is associated with an increase of the filling factor, and is in agreement with a higher retardance value (for instance in Ref. [27]). 
(a)
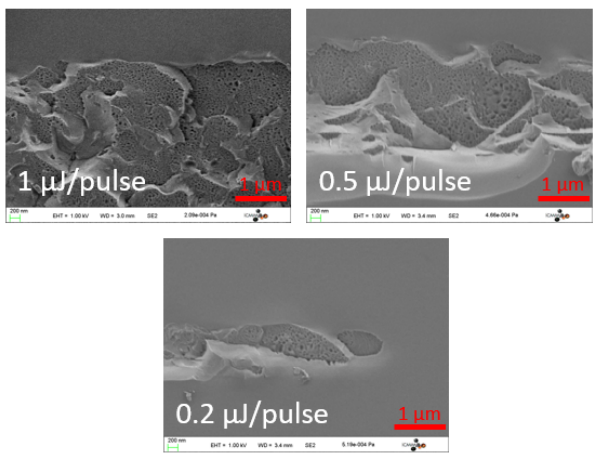

(b)

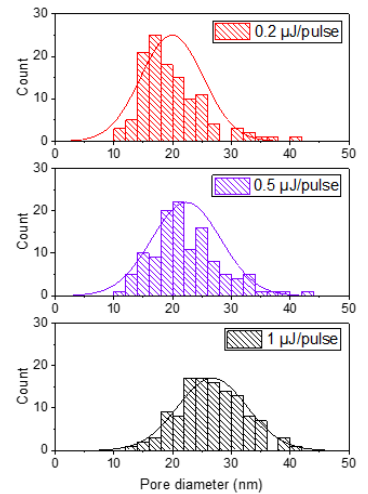

(c)

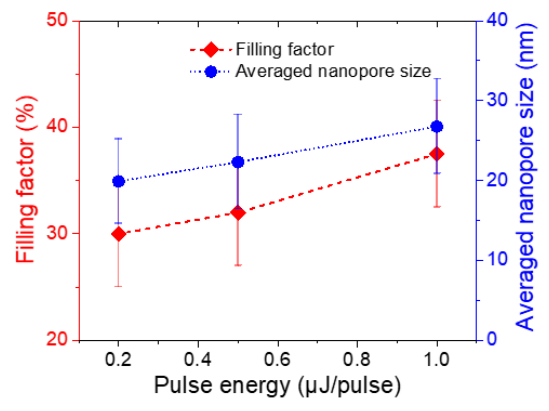

176

Fig. 2 Effect of the laser pulse energy on nanopore size, distribution and filling factor, using Sample S2. (a) Scanning electron micrographs of the porous regions for different pulse energies; (b) Nanopore size diameter distributions with respect to pulse energy; (c) Evolution of the 2D porosity filling factor and the average nanopore size diameter as a function of pulse energy. The lines are here only as guide-to-the-eye

\subsection{Experimental observation of the evolution of the nanopore size in nanogratings}

\section{Experimental observation of the decrease in nanopores size in annealing process}

Finally, the The evolution of the nanopores' morphology and size distribution as a function of annealing temperature (for isochronal time steps $\Delta \mathrm{t}$ of 30 minutes, and using sample S3) is reported in Fig. 3. In this experiment, we observe that the nanopore size diameter decreases first as the temperature increases, but appears to plateau at the highest temperature. This effect can be interpreted from the results given by the R-P equation, and is discussed in the following section. On the other hand, we observe a diminution of $F F_{2 D}$ that is characteristic of a decrease in the birefringence response (hence the measured retardance). Other SEM micrograph analysis, not presented here, also showed that both planes' thickness and spacing between planes were independent of temperature. 
(a)
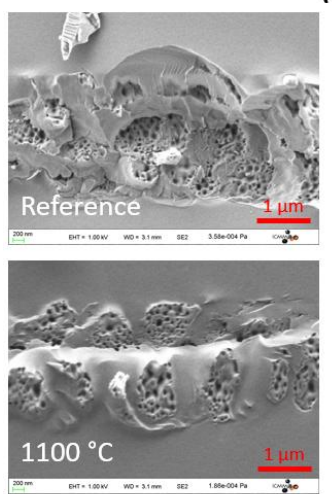

(b)

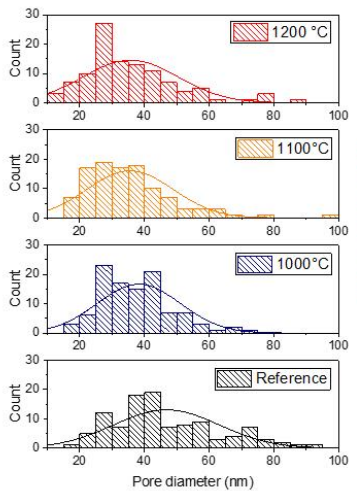

(c)

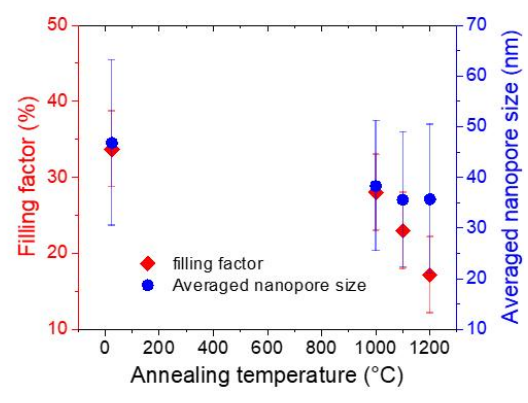

195

Fig. 3 Effect of thermal annealing on nanopore size, distribution and filling factor, using Sample S3. (a) Scanning electron micrographs of the porous regions for different annealing temperatures $(\Delta t=30$ minutes); (b) nanopore size diameter distributions with respect to annealing temperature; (c) Evolution of the 2D porosity filling factor and the average nanopore size diameter as a function of annealing temperature

\subsection{Understanding thermal stability of nanopores using the Rayleigh Plesset equation}

Simulation study of the decrease in nanopores size in annealing process by the RayleighPlesset equation

Thermal stability of Type II nanogratings inscribed in various glasses (silica and silicate glasses) were investigated and reported in Ref. [8], using step isochronal annealing experiments $\left(\Delta t=30\right.$ minutes, $\left.\Delta T=25^{\circ} \mathrm{C}\right)$. These data are displayed in Fig. 4 , but renormalized relative to the sharp retardance decrease at the highest annealing temperature values that we hypothesized here to be characteristic of the nanopore erasure. It is worth pointing out that in order for each data point to be independent to the other, the criterion

$\left(\Delta t \times k_{0}\right)^{-(\Delta T / T \max )} \ll<1$ must be fulfilled [28] (with $\mathrm{k}_{0}$ estimated to be around $5 \times 10^{5}$ to $5 \times 10^{7}$ $\mathrm{s}^{-1}$ for nanogratings erasure in silica [29]). In this work, this criterion is not satisfied and therefore the thermal stability in this condition is expected to be slightly underestimated with respect to a stability curve having independent data points. In this paper only the final erasure step of the measured retardance $R(\Delta t, T)$ is investigated. Following the same experimental conditions as in the experiment, the Rayleigh-Plesset (R-P) equation was used to simulate the nanopore erasure, and the evolution of the retardance was calculated based the nanopore size diameter, the filling factor FF and using the form birefringence equations 
219 (Equations 2, 3, and 4) shown above. Here we assume that the nanolayers thickness $(\delta)$, the 220 average spacing $(\Lambda)$, and the interlayer thickness $(\Lambda-\delta)$, and the laser track length $(L)$ did not 221 change with the annealing steps in agreement with our SEM observations. The comparison 222 between experimental data and the simulated data (using R-P equation) are reported in Fig. 223 4. It is worth pointing out that the R-P equation computed here is for a single initial 224 nanopore size diameter. However, as was previously discussed, the nanopore size diameter 225 is distributed in a fairly wide interval, depending on the laser writing conditions but also 226 with the chemical composition [6, 30]. However, as a first approximation, one can note that 227 evolution of the simulated retardance, related to the erasure of the nanopores using the R-P 228 equation, agrees rather well with the experimental data. This is indicative that viscosity is a 229 first order property in the erasure mechanism of nanogratings and their associated 230 birefringence in a high temperature regime.

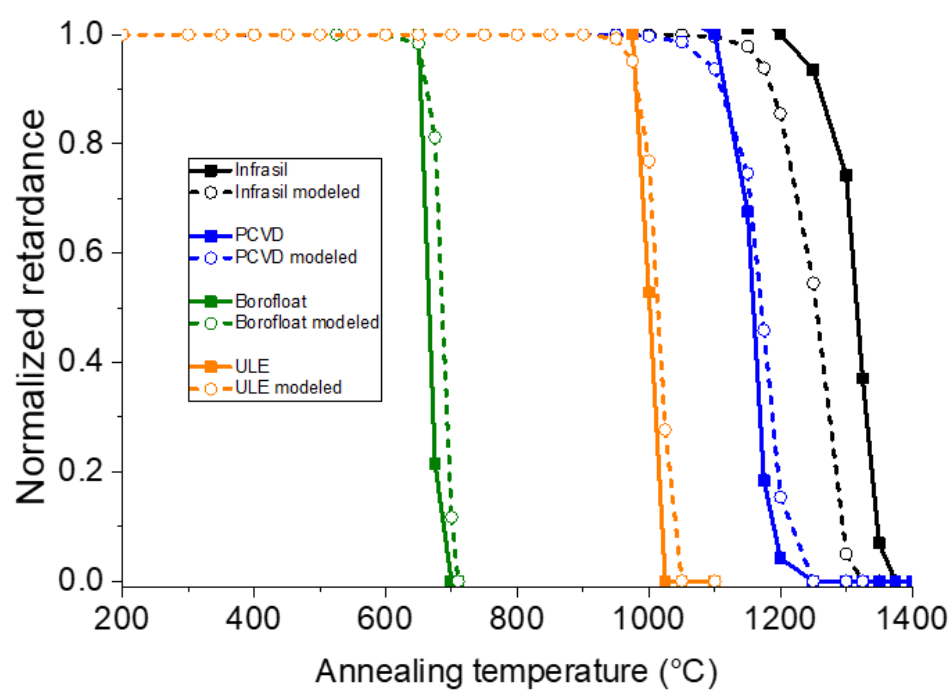

Fig. 4 Evolution of the normalized retardance during step isochronal annealing experiments for various glasses (taken and re-normalized from Ref. [8]) compared with the Rayleigh-Plesset (R-P) equation used to simulate the nanopores' erasure. Conditions used in the simulation: $\Delta P=0 \mathrm{~Pa} ; S=0.3 \mathrm{~J} \mathrm{~m}^{-2} ; \rho=2200 \mathrm{~kg} \mathrm{~m}^{-3}$; Initial nanopore radius used during calculation: $R(t=0)=25 \mathrm{~nm} ; \eta(\mathrm{T})$ fitted using a VTF law for each glass (Infrasil, PCVD, Borofloat33, and ULE) The effects of both nanopore size diameter and surface energy $S$ (from R-P equation) are investigated and reported in Fig. 5 ( $\mathrm{a}$ and b). As the initial nanopore size diameter is increased or the glass surface energy is decreased, the annealing curves shift to higher temperatures. With a $100 \mathrm{~nm}$ nanopore diameter change, thermal stability curve is expected 
to shift by $\square 100^{\circ} \mathrm{C}$ (for a value of $R=0.5$ ). In contrast, the surface energy plays a less

243 significant role in the process since a large change of surface energy $\left(\Delta S=0.4 \mathrm{~J} \mathrm{~m}^{-2}\right)$ shifts 244 only by $\square 50{ }^{\circ} \mathrm{C}$ the annealing curves. The discrepancy between the experimental data and 245 the model observed in Fig. 4 for the Infrasil301 could potentially be attributed to a variation 246 of surface energy, although further investigations would be necessary to verify this 247 hypothesis.

(a)

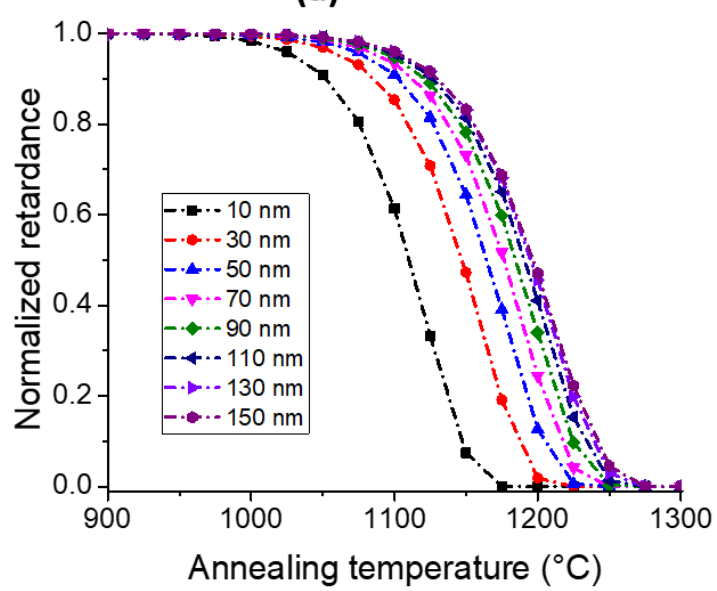

(b)

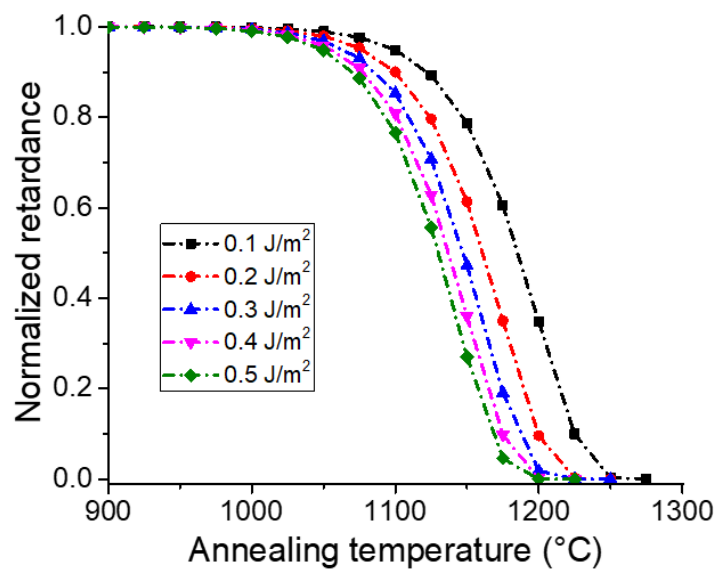

Fig. 5 Evolution of the Retardance (only taking into account the evolution of the nanopores) for step isochronal annealing experiments $(\Delta t=30$ minutes, $\Delta T=25$ ${ }^{\circ} \mathrm{C}$ ) for SuprasilCG glass simulated by Rayleigh-Plesset (R-P) equations. (a) Effect of initial nanopore size diameter (from $10 \mathrm{~nm}$ to $150 \mathrm{~nm}$ ), with a constant value of $S=0.3 \mathrm{~J} \mathrm{~m}^{-2}$. (b) Effect of surface energy $S$ (from $0.1 \mathrm{~J} \mathrm{~m}^{-2}$ to $0.5 \mathrm{~J} \mathrm{~m}^{-2}$ ), with a constant value of $R(t=0)=15 \mathrm{~nm}$

\section{Discussion}

In the previous section we have shown that the thermal stability of nanogratings 258 (evaluated though optical retardance measurements) induced by IR fs-laser (so called Type 259 II regime) is not only a function of the silicate glass chemical composition but also of the 260 nanogratings morphology. The use of various laser-writing parameters, including writing 261 speed (hence linear pulse density) and pulse energy, directly influence the distribution and 262 the size of the nanopores within the porous nanolayers. A direct consequence of this is the change in the thermal stability of optical devices fabricated by Type II fs-laser writing. As

264 an example, the average refractive index changes along the "fringes" of a Type II fiber 
265 Bragg grating (such as the ones in Ref.[2]) would be equal to $\left(n_{e}+n_{o}\right) / 2$. In contrast 266 polarizing based devices like radial-azimuthal polarization converter [31], achromatic 267 polarization rotator [32], fs-laser induced waveplates [13, 33] or 5D "eternal" optical 268 memories [3] are based on the optical retardance amplitude and thus proportional to $\left(n_{e}\right.$ $269 n_{o}$ ). Hence, the optical response of these devices at high temperatures would be related to 270 the magnitude of birefringence induced by the overall porous structure, and among which 271 the nanopore size plays an important role.

272 Additionally, the chemical composition of the glass material has a critical role in the 273 erasure of the nanopores, as its viscosity behavior is found to mostly dictate the overall 274 nanopores' erasure, as shown from the results using R-P equation. For instance, low OH 275 and $\mathrm{Cl}$ impurity-containing materials, such as Infrasil301 silica, present high viscosity at 276 high temperatures, which is expected to promote thermal stability of the nanopores. The 277 differences observed in optical properties, here in term of linear birefringence, are in fairly 278 good agreement between experimental and simulated data using the R-P equation as shown 279 in Fig. 4.

280 From Fig. 1, the average nanopore diameter increases from $53 \mathrm{~nm}$ to $77 \mathrm{~nm}$ between 281 low and high writing speeds $\left(0.01 \mathrm{~mm} \mathrm{~s}^{-1}\right.$ to $\left.10 \mathrm{~mm} \mathrm{~s}^{-1}\right)$, and the steep decay of the 282 associated isochronal annealing curves is shifted by $\square 15^{\circ} \mathrm{C}$ to higher temperatures (Ref. 283 [8]). By using the R-P equation with the experimental conditions described in the same 284 reference, a shift of $\square 15^{\circ} \mathrm{C}$ is also predicted.

285 In Fig. 3c, we observe that, as the irradiated glass is progressively annealed, the 286 nanogratings filling factor $F F$ decreases while the averaged nanopore size remains nearly 287 constant. This can be explained on the basis of the R-P equation, where we observe that 288 small nanopores being erased more quickly than the larger ones (see Fig. 5a). As a result, 289 the fully erased small nanopores are not included in the counting to determine the average 290 nanopore size-diameter. This has an effect of shifting up the average nanopore size diameter 291 to larger values. However, the filling factor still decreases as nanopores are progressively 292 erased, which are in agreement with the observation of a decrease in retardance. One a 293 more speculative note and based on results from Fig. 5b, a possible way to further promote 294 thermal stability could be by minimizing the glass surface energy.

295 As was mentioned in the introduction, it is worth pointing out that the shape of the 296 nanopore is rather ellipsoidal than spherical, as was brought to evidence in Ref. [20] using 297 small angle X-ray scattering (SAXS) measurements. In our conditions and to reveal the 
porosity of the nanopore layers, the sample are cleaved along the short axis direction and placed under an SEM for observation. It is this axis that will determine the kinetics of erasure of the nanopores (based on R-P equation) as the long axis of the nanopores will take

301 a longer time to erase. Although the shape of the annealing curve is expected to be slightly modified, using an ellipsoidal geometry to model the dynamics of the nanopore erasure should not be drastically different with respect to a spherical geometry.

Type III regime, associated with the formation of nanovoids (typically in the 100s $\mathrm{nm}$ to $1 \mu \mathrm{m}$ diameter range [34-36]), is known to be slightly more stable that type II nanogratings, provided that associated stress and densified shell surrounding the nanovoids can be stabilized. From this work, the larger size of the nanopores is expected to be a principal contributor to this higher thermal stability. Hence, in the realm of high thermal stability devices, one would want to maximize the nanopore size diameter. Additionally, a single void structural modification induced by femtosecond laser may be advantageous over nanopores as one would not have to take into account a nanopore size distribution, which may ease thermal stability predictions. Finally, the viscous behavior of the glass material will ultimately set the upper limit of the thermal stability of the device. Hence the current interest is to develop high viscosity glasses and optical fibers [1,37].

\section{Conclusions}

In this work, the effect of glass composition and laser parameters upon fs-laser 317 direct writing in the Type II regime (formation of nanogratings), are reported in silica and 318 silicate glasses. The evolution of nanopores diameter as a function of laser writing speed, 319 pulse energy, and temperature (through step isochronal annealing) is investigated and discussed. The Rayleigh-Plesset (R-P) equation is then used to compute the collapse of such nanopores as a function of glass chemical composition and with varying initial nanopore size diameters or surface energy values. The R-P equation shed light on the major role played by the temperature dependence of the glass viscosity on the thermal stability of

324 fs-induced Type II modifications. The viscous behavior of the glass, at high temperature, is 325 the major parameter that drives the nanogratings erasure and related form birefringence. 326 The initial nanopore size diameter is also found to play a significant role in the overall thermal stability of the fs-laser induced modified region. On a final note, this work aims to serve as a roadmap for the development of components stable at high temperatures, by 
329 mean of both glass property tailoring (e.g., viscosity, surface energy) and the mastering of

330 laser-induced structural modifications (such as the formation of large nanopores or voids).

$331 \quad$ Funding

332 The authors would like to acknowledge the following institutions for funding their research:

333 Agence Nationale pour la Recherche, FLAG/IR project, grant number ANR-18-CE08-

334 0004-01 and CNRS Défi Instrumentation aux Limites, Ultrabragg project.

335 Conflicts of Interest

336 The authors declare no conflict of interest.

337 Availability of data and material (Not applicable)

338 Code availability (Not applicable)

\section{References}

340 1. Cavillon M, Lancry M, Poumellec B, et al (2019) Overview of high temperature 341 fibre Bragg gratings and potential improvement using highly doped aluminosilicate glass optical fibres. J Phys Photonics 1:042001(1)-042001(12). https://doi.org/10.1088/2515-7647/ab382f

344 2. Mihailov SJ, Grobnic D, Hnatovsky C, et al (2017) Extreme Environment Sensing Using Femtosecond Laser-Inscribed Fiber Bragg Gratings. Sensors 17:.

347 3. Zhang J, Gecevičius M, Beresna M, Kazansky PG (2014) Seemingly unlimited lifetime data storage in nanostructured glass. Phys Rev Lett 112:1-5.

350 4. Shimotsuma Y, Kazansky PG, Qiu J, Hirao K (2003) Self-organized nanogratings in 351 glass irradiated by ultrashort light pulses. Phys Rev Lett 91:247405(1)-247405(4). https://doi.org/10.1103/PhysRevLett.91.247405

353 5. Richter S, Miese C, Döring S, et al (2013) Laser induced nanogratings beyond fused 354 silica - periodic nanostructures in borosilicate glasses and ULE ${ }^{\mathrm{TM}}$. Opt Mater Express 3:1161-1166. https://doi.org/10.1364/OME.3.001161 
356 6. Lancry M, Zimmerman F, Desmarchelier R, et al (2016) Nanogratings formation in multicomponent silicate glasses. Appl Phys B Lasers Opt 122:1-8. https://doi.org/10.1007/s00340-016-6337-8

359 7. Bricchi E, Kazansky PG (2006) Extraordinary stability of anisotropic femtosecond 360 direct-written structures embedded in silica glass. Appl Phys Lett 88:2-4. https://doi.org/10.1063/1.2185587

8. Wei S-E, Wang Y, Yao H, et al (2020) Thermal stability of type II modifications by IR femtosecond laser in silica-based glasses. Sensors 20:1-14. https://doi.org/10.3390/s20030762

9. Witcher JJ, Reichman WJ, Fletcher LB, et al (2013) Thermal annealing of femtosecond laser written structures in silica glass. Opt Mater Express 3:502-510. https://doi.org/10.1364/OME.3.000502

10. Lu J, Yang M, Wang DN, et al (2009) Fiber Bragg gratings with enhanced thermal stability by residual stress relaxation. Opt Express 17:19785-19790. https://doi.org/10.1364/oe.17.019785

11. Thomson RR, Gillet P, Mukherjee S, et al (2016) Stress-state manipulation in fused silica via femtosecond laser irradiation. Optica 3:1285. https://doi.org/10.1364/optica.3.001285

12. Bhardwaj VR, Corkum PB, Rayner DM, et al (2004) Stress in femtosecond-laserwritten waveguides in fused silica. Opt Lett 29:1312-1314.

377 13. Tian J, Yao H, Cavillon M, et al (2020) A comparison between nanogratings-based and stress-engineered waveplates written by femtosecond laser in silica.

380 14. Lancry M, Poumellec B, Canning J, et al (2013) Ultrafast nanoporous silica 381 formation driven by femtosecond laser irradiation. Laser Photonics Rev 7:953-962. 382 https://doi.org/10.1002/lpor.201300043

383 15. Canning J, Lancry M, Cook K, et al (2011) Anatomy of a femtosecond laser 
processed silica waveguide. Opt Mater Express 1:998-1008. https://doi.org/10.1364/ome.1.000998

16. Berger AJ, Wang Y, Sammeth DM, et al (1995) Aqueous Dissolved Gas Measurements Using Near-Infrared Raman Spectroscopy. Appl Spectrosc 49:11641169. https://doi.org/10.1366/0003702953965047

17. Zimmermann F (2017) Ultrashort pulse induced nanostructures in transparent materials. Friedrich-Schiller-Universitat Jena

391 18. Desmarchelier R, Poumellec B, Brisset F, et al (2015) In the Heart of Femtosecond

19. Rudenko A, Colombier JP, Itina TE (2018) Nanopore-mediated ultrashort laserinduced formation and erasure of volume nanogratings in glass. Phys Chem Chem Phys 20:5887-5899. https://doi.org/10.1039/c7cp07603g

20. Richter S, Plech A, Steinert M, et al (2012) On the fundamental structure of femtosecond laser-induced nanogratings. Laser Photonics Rev 6:787-792. https://doi.org/10.1002/lpor.201200048

21. Sakakura M, Lei Y, Wang L, et al (2020) Ultralow-loss geometric phase and polarization shaping by ultrafast laser writing in silica glass. Light Sci Appl 9:1-10. https://doi.org/10.1038/s41377-020-0250-y

22. Plesset MS (1949) The Dynamics of Cavitation Bubbles. J Appl Mech 16:277-282. https://doi.org/10.1080/15435075.2018.1431546

23. Brennen CE (1995) Cavitation and bubble dynamics. Oxford University Press

24. Boyd K, Ebendorff-Heidepriem H, Monro TM, Munch J (2012) Surface tension and

25. Markel VA (2016) Introduction to the Maxwell Garnett approximation: tutorial. J 
412 27. Lancry M, Canning J, Cook K, et al (2016) Nanoscale femtosecond laser milling and 413 control of nanoporosity in the normal and anomalous regimes of $\mathrm{GeO}_{2}-\mathrm{SiO}_{2}$ glasses. 414 Opt Mater Express 6:321. https://doi.org/10.1364/OME.6.000321

415 28. Poumellec B, Lancry M (2015) Kinetics of Thermally Activated Physical Processes 416 in Disordered Media. Fibers 3:206-252. https://doi.org/10.3390/fib3030206

417 29. Gecevičius M (2015) Polarization Sensitive Optical Elements By Ultrafast Laser 418 Nanostructuring of Glass. University of Southampton

419 30. Zimmermann F, Lancry M, Plech A, et al (2016) Femtosecond laser written 420

31. Beresna M, Gecevičius M, Kazansky PG, Gertus T (2011) Radially polarized optical vortex converter created by femtosecond laser nanostructuring of glass. Appl Phys Lett 98:1-4. https://doi.org/10.1063/1.3590716

32. Desmarchelier R, Lancry M, Gecevicius M, et al (2015) Achromatic polarization rotator imprinted by ultrafast laser nanostructuring in glass. Appl Phys Lett 107:181111(1)-18111(4). https://doi.org/10.1063/1.4934866

33. Gecevičius M, Beresna M, Kazansky PG (2013) Polarization sensitive camera by femtosecond laser nanostructuring. Opt Lett 38:4096-4099. https://doi.org/10.1364/ol.38.004096

431 34. Williams RJ, Krämer RG, Nolte S, et al (2013) Detuning in apodized point-by-point fiber Bragg gratings: insights into the grating morphology. Opt Express 21:2685426867. https://doi.org/10.1364/oe.21.026854

35. Glezer EN, Milosavljevic M, Huang L, et al (1996) 3-D Optical Storage Inside Transparent Materials. Opt Lett 21:2023-2026

36. Gamaly EG, Juodkazis S, Nishimura K, et al (2006) Laser-matter interaction in the 438 bulk of a transparent solid: Confined microexplosion and void formation. Phys Rev B - Condens Matter Mater Phys 73:1-15. https://doi.org/10.1103/PhysRevB.73.214101 
440 37. Wang Y, Wei S, Yuta RC, et al (2020) Femtosecond laser direct writing in SiO2 441 Al2O3 binary glasses and thermal stability of Type II permanent modifications. J 442 Am Ceram Soc 1-9. https://doi.org/10.1111/jace.17164

443 